\title{
Effect of KTH Integrated Nursing Intervention on Self-efficacy and Lifestyle in Patients with Breast Cancer Chemotherapy
}

\section{Mei Wang, Yang Yang, Shiqing Sun, Fangcao He, Congcong Li}

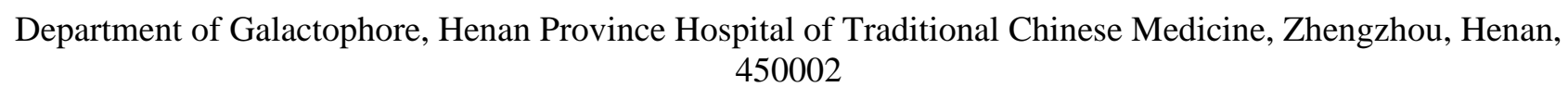

Keywords: breast cancer; Chemotherapy; KTH integrated nursing intervention; self-efficacy; lifestyle

\begin{abstract}
To investigate the effect of KTH integrated nursing intervention in patients with breast cancer chemotherapy, thirty-nine breast cancer patients undergoing chemotherapy in our hospital were randomized according to the random number table method. 39 patients in the control group were treated with routine nursing intervention. 40 patients in the observation group were treated with KTH integrated nursing intervention on the basis of routine nursing care. Pre- and post-intervention GSES scores, lifestyle (sports, self-realization, nutrition, interpersonal relationships, health responsibilities, stress management) scores, and cancer-induced fatigue (RPFS) scores. The GSES score was higher in the two groups after the intervention for 3 months than before the intervention, and the GSES score was higher in the observation group after 3 months of intervention than in the control group $(\mathrm{P}<0.05)$. After 3 months of intervention, the observation group sports, self-realization, nutrition, The treatment scores of interpersonal relationships, health responsibilities, and stress were higher than those in the control group $(\mathrm{P}<0.05)$; the RPFS scores were lower than the pre-intervention 3 months after the intervention and the RPFS scores were lower in the observation group than in the control group after 3 months of intervention $(\mathrm{P}) .<0.05)$. $\mathrm{KTH}$ integrated nursing intervention can effectively improve the self-efficacy of patients with breast cancer chemotherapy, improve their lifestyle, and relieve the degree of cancer-induced fatigue.
\end{abstract}

\section{Introduction}

Breast cancer is a malignant tumor with a high incidence rate. According to statistics, up to 7\% to $10 \%$ of women with systemic malignancies are breast cancers, ranking second in female malignant tumors, and at a rate of $2 \%$ to $3 \%$ per year. Growth has become a major malignancy that threatens women's life and health [1]. Chemotherapy is still the main treatment method for breast cancer. It can control the metastasis and infiltration of cancer cells to a certain extent to control the deterioration of the disease. However, during chemotherapy and after chemotherapy, patients are prone to develop cancer-related fatigue and affect the normal progress of chemotherapy. Some patients even break down. Chemotherapy leads to aggravation of his condition. The occurrence of cancer-related fatigue is closely related to the behavioral and self-management skills of patients. Some scholars have pointed out that certain health education can prompt patients to consciously correct their bad life habits and establish a healthy lifestyle [2]. KTH Integrated Nursing Intervention is an integrated health education concept that integrates the three models of cross-theoretical model, knowledge and belief model and health belief model. It provides patients with comprehensive, continuous, and targeted disease information from multiple angles, satisfying the patient's needs. The demand for disease knowledge makes it possible to recognize the influence of good living habits on the disease, consciously develop good living habits, and promote disease recovery. In this study, KTH integrated care intervention was applied to breast cancer patients with chemotherapy and achieved significant results. The following is reported. 


\section{Objects and Methods}

Subjects 79 breast cancer patients undergoing chemotherapy in our hospital from February 2015 to November 2017 were selected and grouped according to the random number table method. The control group consisted of 39 patients, aged 37 to 62 years, mean age (48.04 \pm 8.20$)$. Years; duration

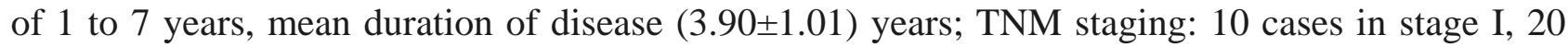
cases in stage IIa, and 9 cases in stage IIb. Observation group 40 cases, aged 36 to 61 years old, mean age (48.15 \pm 8.09$)$ years; duration of 1 to 6 years, mean duration of disease (3.20 \pm 1.30$)$ years; TNM staging: 11 cases in stage I, 21 cases in stage IIa, stage IIb 8 cases. Comparing the baseline data between the two groups, there was no significant difference $(P>0.05)$, and this study was approved by the Ethics Committee of our institute.

Inclusion Criteria (1) Conforms to "Surgery" diagnostic criteria for breast cancer [3]; (2) Chemotherapy is performed; (3) Both are elementary school and above; (4) Knowing this study and voluntarily signing informed consent book.

Exclusion criteria (1) Heart, brain, lung and other vital organs failure; (2) with immune system disorders; (3) with sinus bradycardia; (4) coexist with other malignancy disorders; (5) merger Blood system disorders; (6) Associated with syphilis or active tuberculosis or other infectious diseases; (7) Severe cognitive impairment.

Control group Routine nursing interventions: (1) Simply introduce patients to breast cancer chemotherapy. (2) Pay close attention to the patient's condition and notify the doctor in time if any signs of complications appear. (3) Instruct patients to eat more crude fruit such as fresh fruits and vegetables, and drink plenty of water to aid hydration.

Observation group To implement KTH integrated nursing intervention on the above basis: (1) Form a group. The KTH Integrated Nursing Intervention Team is composed of attending physicians, head nurses, and responsible nurses; experienced nursing experts are invited to provide knowledge of the knowledge and belief model $(\mathrm{K})$, cross-theoretical model $(\mathrm{T})$, and health belief model $(\mathrm{H})$ to the team members. Operation skill training, after the training, the team members will be assessed, and the appraisal can be performed after the appraisal. (2) Make a plan. According to the level of education of patients and their families, and the acceptance of new knowledge, the model of health beliefs, cross-theoretical models, and knowledge and belief models were integrated, and breast cancer chemotherapy intervention programs (intervention methods and procedures) were developed. (3) Implementation. Nursing staff through one-on-one communication with patients and their families to assess their current status of knowledge and understanding, a comprehensive understanding of the patient's knowledge of breast cancer and chemotherapy knowledge, illness, lifestyle, psychological status, family status, chemotherapy compliance, etc. According to the cross-theoretical model, the state of the patients' knowledge and belief is divided into different levels, targeted nursing measures are implemented according to the state of knowledge, belief, and health belief patterns; and breast cancer health knowledge brochures with illustrations and illustrations are distributed to patients (the manual contains the causes and conditions of breast cancer. Progress, clinical manifestations, risk factors, and current treatment measures, etc.) 1 time/month for detailed knowledge of breast cancer knowledge, $1 \mathrm{~h} /$ time; rolling breast cancer related knowledge and health education videos in the lobby; weekly 1 patient Assessment of breast cancer-related knowledge, according to the assessment results for their unabated areas to strengthen health education; one-on-one guidance of patients with scientific, rational drug use, daily supervision 1 or 2 times; inform patients of the importance of continuing physical exercise , and guide and urge patients to exercise, not less than 3 times a week, each time about 30 min. (4) Establish a patient communication platform. Establish WeChat and QQ breast cancer patient exchange groups. Exchange groups are managed by full-time nurses. We regularly provide breast cancer related knowledge, the latest treatment and nursing, and activity arrangements through WeChat, QQ platform, to provide patients with free and timely health advice. Answer patient questions; encourage patients to communicate, share, and communicate within the group. All interventions for 3 months. 
Observed indicators (1) The general self-efficacy scale (GSES) was used to evaluate self-efficacy before and after 3 months of intervention. The higher the score, the higher the self-efficacy [4]. (2) Using the Health Promotion Lifestyle Rating Scale (HPLS-II) to assess the lifestyle of the two groups prior to intervention and after 3 months of intervention, including treatment of physical activity, self-realization, nutrition, interpersonal relationships, health responsibilities, and stress 6 On the other hand, the higher the score, the better the lifestyle improvement [5]. (3) The cancer-causing fatigue assessment scale (RPFS) was used to assess the degree of cancer-related fatigue before and after intervention for 3 months. The higher the score, the more severe the fatigue is [6].

Statistical analysis SPSS20.0 analysis of data processing, $( \pm \mathrm{s})$ said the measurement data, $\mathrm{t}$ test, the difference was statistically significant standard $\mathrm{P}<0.05$.

\section{Results}

GSES score After 3 months of intervention, the GSES scores of the two groups were higher than those before the intervention, and the observation group was higher than the control group $(\mathrm{P}<0.05)$. See Table 1.

Table 1 Comparison of two groups of GSES scores ( \pm s, points)

\begin{tabular}{lccccc}
\hline \multicolumn{1}{c}{ Groups } & Num & $\begin{array}{c}\text { Before } \\
\text { intervention }\end{array}$ & $\begin{array}{c}\text { After 3 } \\
\text { months of } \\
\text { intervention }\end{array}$ & $t$ & $P$ \\
\hline $\begin{array}{l}\text { Observation } \\
\text { group }\end{array}$ & 40 & $15.10 \pm 2.60$ & $30.40 \pm 4.70$ & 18.016 & 0.000 \\
Control & 39 & $15.53 \pm 2.57$ & $20.60 \pm 5.25$ & 5.417 & 0.000 \\
group & $/$ & & 8.746 & $/$ & $/$ \\
$t$ & $/$ & & 0.000 & $/$ & $/$ \\
$P$ & & &
\end{tabular}

Lifestyle The scores of sports, self-realization, nutrition, interpersonal relationships, health responsibilities, and stress were higher in the observation group after 3 months of intervention than in the control group $(\mathrm{P}<0.05)$. See Table 2.

Table 2 Comparison of two groups of lifestyle index scores ( \pm s, points)

\begin{tabular}{|c|c|c|c|c|c|c|c|c|}
\hline Time & Groups & Num & Sports & nutrition & Health duties & Self-realization & $\begin{array}{l}\text { Human } \\
\text { relations }\end{array}$ & $\begin{array}{l}\text { Pressure } \\
\text { treatment }\end{array}$ \\
\hline \multirow{4}{*}{$\begin{array}{l}\text { Before } \\
\text { intervention }\end{array}$} & $\begin{array}{l}\text { Observati } \\
\text { on group }\end{array}$ & 40 & $2.40 \pm 0.50$ & $2.47 \pm 0.73$ & $2.50 \pm 0.50$ & $2.30 \pm 0.50$ & $2.50 \pm 0.60$ & $2.45 \pm 0.75$ \\
\hline & $\begin{array}{l}\text { Control } \\
\text { group }\end{array}$ & 39 & $2.35 \pm 0.75$ & $2.54 \pm 0.80$ & $2.60 \pm 0.41$ & $2.35 \pm 0.45$ & $2.60 \pm 0.50$ & $2.70 \pm 0.61$ \\
\hline & $t$ & / & 0.350 & 0.406 & 0.971 & 0.467 & 0.804 & 1.623 \\
\hline & $P$ & / & 0.728 & 0.686 & 0.335 & 0.642 & 0.424 & 0.109 \\
\hline \multirow{4}{*}{$\begin{array}{l}\text { After } 3 \\
\text { months } \\
\text { of } \\
\text { intervention }\end{array}$} & $\begin{array}{l}\text { Observati } \\
\text { on group }\end{array}$ & 40 & $3.01 \pm 0.78$ & $3.30 \pm 0.60$ & $3.65 \pm 0.30$ & $3.65 \pm 0.35$ & $3.40 \pm 0.50$ & $3.55 \pm 0.42$ \\
\hline & $\begin{array}{l}\text { Control } \\
\text { group }\end{array}$ & 39 & $2.60 \pm 0.70$ & $2.80 \pm 0.70$ & $2.90 \pm 0.70$ & $2.70 \pm 0.50$ & $2.95 \pm 0.24$ & $2.94 \pm 0.50$ \\
\hline & $t$ & / & 2.457 & 3.412 & 6.217 & 9.804 & 5.078 & 5.877 \\
\hline & $P$ & / & 0.016 & 0.001 & 0.000 & 0.000 & 0.000 & 0.000 \\
\hline
\end{tabular}

RPFS scores After 3 months of intervention, the RPFS scores of the two groups were lower than 
before intervention, and the observation group was lower than the control group $(\mathrm{P}<0.05)$. See Table 3.

Table 3 Comparison of two groups of RPFS scores ( \pm s, points)

\begin{tabular}{lccccc}
\hline Groups & Num & $\begin{array}{c}\text { Before } \\
\text { intervention }\end{array}$ & $\begin{array}{c}\text { After 3 } \\
\text { months of } \\
\text { intervention }\end{array}$ & $t$ & $P$ \\
\hline $\begin{array}{l}\text { Observation } \\
\text { group }\end{array}$ & 40 & $114.80 \pm 7.68$ & $\begin{array}{c}77.50 \pm 12.9 \\
1\end{array}$ & 15.704 & 0.000 \\
$\begin{array}{l}\text { Control } \\
\text { group }\end{array}$ & 39 & $115.90 \pm 6.37$ & $\begin{array}{c}105.82 \pm 7.6 \\
9\end{array}$ & 6.304 & 0.000 \\
$t$ & $/$ & 0.692 & 11.807 & $/$ & $/$ \\
$P$ & $/$ & 0.491 & 0.000 & $/$ & $/$ \\
\hline
\end{tabular}

\section{Discussion}

Chemotherapy can kill cancer cells, reduce the tumor stage of patients, and prolong their survival time. It is one of the main measures for the treatment of breast cancer, but it also causes a series of adverse reactions. Statistics show that up to $58 \%$ to $94 \%$ of breast cancer patients may experience cancer-related fatigue during chemotherapy, and the incidence of cancer-related fatigue after chemotherapy is also as high as $56 \%$ to $95 \%$, which is not conducive to the smooth implementation of chemotherapy [7]. Individual behavioral risk factors are closely related to the incidence of cancer-induced fatigue during chemotherapy and after chemotherapy. By controlling behavioral risk factors and changing the patient's unhealthy lifestyle, the incidence of cancer-induced fatigue can be reduced and the quality of life of patients can be improved.

Some scholars have pointed out that the improvement of self-efficacy of cancer patients is the key to change their behavior, solve the prognosis of rehabilitation and disease adaptation [8]. Therefore, taking reasonable and effective measures to enhance the self-efficacy of breast cancer chemotherapy patients is of great significance in changing their lifestyle and reducing the incidence of cancer-related fatigue. Routine nursing interventions are limited to observation of illnesses, dietary guidance, etc., and lack of targeted health education on breast cancer and chemotherapy. It is difficult to meet the needs of patients for disease and related treatment knowledge, leading to their inability to objectively and comprehensively understand the disease and self-efficacy. Feeling low, then affecting their lifestyle. The study found that after 3 months of intervention, the GSES scores were higher in the two groups than before the intervention, and the GSES score was higher in the observation group after 3 months of intervention than in the control group $(\mathrm{P}<0.05)$. It can be seen that the routine nursing intervention can improve the patient's self to a certain degree. The efficacy, but far less than the observation group, KTH integrated care intervention can significantly enhance the self-efficacy of patients with breast cancer chemotherapy. This may be related to the following factors: (1) KTH integrated health intervention integrates the advantages of the three modes of knowledge and communication, health beliefs, and cross-theoretical models to make the formulated health education program more scientific and rational. The implementation of missions provides a theoretical basis; (2) By assessing the status of patients' knowledge and understanding, and dividing them into different levels according to the status of patients' knowledge and belief under the cross-theory model, the entire intervention measures are more targeted and implemented by different patients. Personalized health education, focused knowledge on disease knowledge, and video scrolling, and a series of breast cancer knowledge and chemotherapy knowledge presentations can enable patients to recognize the benefits of adopting healthy behaviors, and urge them to conscientiously change their bad habits, with a positive and optimistic attitude. For breast cancer chemotherapy, it can help the smooth progress of chemotherapy and improve the quality of life. This study found that after 3 months of observation group intervention, sports, self-realization, nutrition, interpersonal relationships, health responsibilities, and stress management scores were 
higher than in the control group $(\mathrm{P}<0.05)$. It can be seen that $\mathrm{KTH}$ integrated care intervention can change breast cancer chemotherapy patients. lifestyle. At the same time, the study found that the RPFS score in the observation group significantly decreased after 3 months of intervention and was lower than the control group $(\mathrm{P}<0.05)$, indicating that $\mathrm{KTH}$ integrated care intervention can improve the degree of cancer-related fatigue in patients with breast cancer chemotherapy, which may be due to KTH integration The type of nursing intervention enhanced the patient's self-efficacy, and the improvement of self-efficacy enhanced the belief of the patient in restoring health, conscientiously changed the unhealthy lifestyle, and established a healthy way of life and behavior, thereby alleviating cancer-induced fatigue.

In summary, KTH integrated care intervention can enhance the self-efficacy of breast cancer patients undergoing chemotherapy, change their lifestyle, and relieve cancer-causing fatigue during and after chemotherapy, which is worthy of popularization and application.

\section{Acknowledgements}

Fund Project: Study on the efficacy and mechanism of Shenyan Pill combined with tamoxifen in inhibiting the growth of breast tumor and preventing lung metastasis based on traditional Chinese medicine "Consumption method", 13A360610

\section{References}

[1] Shao Xianqing, Wu Xinhua, Zhang Ziyang, et al. Effects of family synchronization health education on postoperative self-efficacy and functional exercise compliance of breast cancer patients[J]. Cancer Progress, 2017, 15(2): 208-210.

[2] Wu Qingshi, Yuan Huiling, Diao Baoling, et al. Effect of belief-based health education on the selection of PICC chemotherapy in breast cancer patients[J]. International Medicine \& Health Guidance News,2014,20(2):277-280.

[3] Chen Xiaoping, Wang Jianping. Surgery [M]. 8th ed. Beijing: People's Medical Publishing House, 2013:256-260.

[4] Gao Yi, Shi Tieying. Correlation between post-traumatic growth and general self-efficacy and perceived social support in breast cancer patients[J]. Nursing Research, 2017, 31(3): 281-284.

[5] Zhong Qiaoling, Wang Haichun. "In-hospital-out-of-hospital" extended nursing model on the health behaviors and mood of patients undergoing chemotherapy after radical mastectomy[J]. Modern Clinical Nursing, 2016,15(12):50-55.

[6] Cao Yuyao, Song Yi, Chen Fengmin, et al. Effects of aerobic exercise on cancer-induced fatigue in breast cancer patients and related mechanisms[J]. Tianjin Medicine, 2016, 44(4): 401-404.

[7] Qin Yanyan.Effects of informational beliefs,behavior patterns and nursing interventions on cancer-induced fatigue and negative emotions in breast cancer patients[J].Journal of Qilu Nursing,2016,22(4):41-42.

[8] Liang Lixue, Liu Qingping, Yan Cuifang, et al. Effect of knowledge and health education intervention on health knowledge and self-efficacy of gastric cancer patients[J]. China Practical Medicine, 2017, 12(13): 173-175. 